\title{
Nested trellis codes and shaping for the transmitter side-information problem
}

\author{
Baltasar Beferull-Lozano \\ Swiss Federal Inst. of Tech.-EPFL \\ AudioVisual Communications Laboratory \\ CH-1015, Lausanne, Switzerland \\ e-mail: baltasar.beferull@epfl.ch
}

\section{Introduction AND PRELIMINARIES}

The communication channel with transmitter sideinformation [1] is modeled as follows. If $\boldsymbol{X}, \boldsymbol{Y}, \boldsymbol{S}$ and $\boldsymbol{Z}$ are random $N$-dimensional vectors

$$
\boldsymbol{Y}=\boldsymbol{X}+\boldsymbol{S}+\boldsymbol{Z}
$$

where $\boldsymbol{X}$ is the transmit vector signal, $\boldsymbol{Y}$ is the receive vector signal, $\boldsymbol{S}$ is a Gaussian interference vector signal, and $Z$ is a Gaussian noise vector. The interference signal $\boldsymbol{S}$ is non-causally known at the transmitter but not known at the receiver, and the noise $\boldsymbol{Z}$ is independent of $\boldsymbol{X}$ and $\boldsymbol{S}$ and it is not known neither at the transmitter nor at the receiver. The transmit signal must satisfy a power constraint, that is, $\frac{1}{N} E\left[\|\boldsymbol{X}\|^{2}\right] \leq P$. Using random binning arguments, the capacity of this channel is given by ${ }^{1} C=$ $\frac{1}{2} \log \left(\operatorname{det}\left(\boldsymbol{K}_{\boldsymbol{X}}+\boldsymbol{K}_{\boldsymbol{Z}}\right) / \operatorname{det}\left(\boldsymbol{K}_{\boldsymbol{Z}}\right)\right)$ where $\boldsymbol{K}_{\boldsymbol{X}}$ and $\boldsymbol{K}_{\boldsymbol{Z}}$ are the covariance matrices of $\boldsymbol{X}$ and $\boldsymbol{Z}$ respectively. Thus, the effect of the interference $\boldsymbol{S}$ is completely eliminated (by utilizing it through the transmitter side-information) and the capacity of this channel is the same as for the gaussian vector channel without any interference $\boldsymbol{S}$. In [2], a constructive approach for the random binning arguments of [1] was given through the use of nested lattices $\Lambda^{1} / \Lambda^{2}$.

In this paper, we specifically focus on the problem of power shaping and we examine nested constructions based on trellis codes, which build on simple low dimensional lattices. We propose the idea of performing shaping through a coarse lattice (source code) and we also show how this method can actually be combined with shaping through a fine lattice (channel code) so that a joint shaping can be performed by the fine and coarse lattices simultaneously.

\section{SHAPING THROUGH A COARSE LATTICE}

Let $\Lambda^{1} / \Lambda^{3} / \Lambda^{4}$ be a lattice partition chain where $\left|\Lambda^{1} / \Lambda^{3}\right|=$ $2^{n_{q}}$ and $\left|\Lambda^{3} / \Lambda^{4}\right|=2^{n_{s}}$. Let also $\Lambda^{3}=\Lambda_{c_{1}}^{4} \cup \cdots \cup \Lambda_{c_{2} n_{s}}^{4}$ where $\Lambda_{c_{1}}^{4}=\Lambda^{4}$ and $\Lambda_{c_{i}}^{4}$ is the $i$-th coset. We denote by $Q_{\Lambda_{c_{i}}^{4}}$ the nearest neighbor quantizer whose reconstruction points are given by the coset $\Lambda_{c_{i}}^{4}$. We associate with each message $m$ one and only one signal vector $v$ of the constellation composed by the $2^{n_{q}}$ points in $\Lambda^{1} \cap C_{\Lambda^{3}}(\mathbf{0})$, that is, no constellation expansion takes place, as opposed to the trellis precoding method [3]. Let $\left\{\boldsymbol{m}_{i_{1}}, \boldsymbol{m}_{i_{2}}, \cdots, \boldsymbol{m}_{i_{J}}\right\}$ and $\left\{\boldsymbol{s}_{1}, \boldsymbol{s}_{2}, \cdots, \boldsymbol{s}_{J}\right\}$ again denote the sequence of $J$ binary messages to be transmitted and the corresponding sequence of interference vectors. The shaping operation consists of choosing the best sequence $\left\{Q_{\Lambda_{c_{i_{j}}}^{4}}\right\}_{j=1}^{J}$ such that the average power $P$ per dimension is minimized:

$\operatorname{Min}_{\left\{Q_{\Lambda_{c_{i_{j}}}^{4}}\right\}_{j=1}^{J}} P=\frac{1}{N J} \sum_{j=1}^{J}\left\|\left(\boldsymbol{v}_{i_{j}}-\boldsymbol{s}_{j}\right)-Q_{\Lambda_{\boldsymbol{c}_{i_{j}}}^{4}}\left(\boldsymbol{v}_{i_{j}}-\boldsymbol{s}_{j}\right)\right\|^{2}$

${ }^{1} \boldsymbol{S}$ can be also an ergodic process or an arbitrary sequence.

\author{
Suhas Diggavi \\ AT\&T Shannon Labs \\ 180 Park Ave, Florham Park \\ New Jersey, 07932, USA \\ e-mail: suhas@research.att.com
}

The goal we want to achieve is to increase the bit rate while generating a non-uniform distribution of power. The idea is to constrain the choices of the quantizers through the whole sequence by using a $k_{s} / n_{s}$ shaping convolutional code $C_{s}$ where each path will correspond now to a particular sequence of quantizers. Thus, the shaping will be obtained by choosing among all the possible paths in the code $C_{s}$ the path that gives the lowest average power. In this case, each branch of the trellis diagram of the code $C_{s}$ will be associated with a particular quantizer and comming out from each state, there will be only $2^{k_{s}}$ quantizers to choose from. It can be shown that encoding and decoding can be correctly defined by using the concept of syndrome-former $\boldsymbol{H}_{s}^{T}(D)$.

III. JOINT SHAPING WITH FINE AND COARSE LATTICES Let $\Lambda^{1} / \Lambda^{2} / \Lambda^{3} / \Lambda^{4}$ be a lattice partition chain as in Section II and where the nesting $\Lambda^{1} / \Lambda^{2}$ gives shaping through the fine lattice. Now, we jointly shape through the channel and the source lattices by allowing to choose jointly from a sequence of signal points and quantizers. Let $T_{p}$ and $T_{s}$ denote the trellis associated with the convolutional codes $C_{p}$ and $C_{s}$ respectively. Let $T_{p}$ have states $v_{1}, \cdots, v_{2^{b_{p}}}$ and $T_{s}$ have states $\omega_{1}, \cdots, \omega_{2 b_{s}}$. The combination of the two methods is performed through the tensor product $T_{p} \otimes T_{s}$ of the two trellises. The tensor product $T_{p} \otimes T_{s}$ is a trellis with $2^{b_{p}+b_{s}}$ states $v_{n} \otimes \omega_{m}, n=1, \cdots, 2^{b_{p}}, m=1, \cdots, 2^{b_{s}}$. There is a transition between states $v_{n} \otimes \omega_{m}$ and $v_{t} \otimes \omega_{r}$ in $T_{p} \otimes T_{s}$ if and only if there exist transitions between $v_{n}$ and $v_{t}$ in $T_{p}$ and between $\omega_{m}$ and $\omega_{r}$ in $T_{s}$. It is important to note that in the process of encoding and decoding, there is no need to actually implement the trellis $T_{p} \otimes T_{s}$, with $2^{b_{p}+b_{s}}$ states but one only needs to perform the operations for each time slot in two stages, one stage (sub-transition) using $T_{p}$ and the other stage (sub-transition) using $T_{s}$. Again, encoding and decoding operations can be correctly defined.

\section{EXPERIMENTAL RESULTS}

As an example, for the case of $T_{p}=T_{s}=T_{U}$ (Ungerboeck's trellis) with $k_{p}=k_{s}=1, n_{p}=n_{s}=2$ and $b_{s}=b_{p}=2(4$ states) and $\Lambda_{1}=\mathbb{Z}^{2}, \Lambda^{2}=2 \Lambda_{1}, \Lambda^{3}=4 \Lambda_{1}$ and $\Lambda^{4}=8 \Lambda_{1}$, gains around $1 \mathrm{~dB}$ (depending on the decoding window size) are obtained over the baseline system.

\section{REFERENCES}

[1] M. Costa, "Writing on dirty paper," IEEE Trans. on Information Theory, vol. 29, no. 3, pp. 439-441, 1983.

[2] R. Zamir, S. S. (Shitz), and U. Erez, "Nested linear/lattice codes for structured multiterminal binning," IEEE Trans. on Information Theory, vol. 48, no. 6, pp. 1250-1276, 2002.

[3] M. V. Eyuboglu and G. D. Forney, "Trellis precoding: combined coding,precoding and shaping for intersymbol interference channels," IEEE Trans. on Inf. Th., vol. 38, no. 2, pp. 301-313, 1992. 